\title{
INTENSE RIGIDITY OF THE ARMS DUE TO ISOLATION OF MOTONEURONES BY A SPINAL TUMOUR
}

\author{
BY
GEOFFREY RUSHWORTH, W. A. LISHMAN, J. TREVOR HUGHES, and D. R. OPPENHEIMER \\ From the Neurological Research Unit, Churchill Hospital, Oxford, and the Departments of \\ Neurology and Neuropathology, Radcliffe Infirmary, Oxford
}

In man, persisting muscular rigidity* is usually associated with severe brain damage, as in the decerebrate state and various extrapyramidal disorders. It may also be seen following tetanus. More rarely, cervical cord damage may cause rigidity of the muscles of the arms and shoulder girdle instead of the more usual spasticity. Rigidity of spinal origin is poorly recognized, but recently Penry, Hoefnagel, van den Noort, and Denny-Brown (1960) have reviewed six cases from the literature and have added three examples of their own, including a brief account of the present patient during life. This patient developed intense rigidity in the arms as a result of a spinal neoplasm, and the clinical evolution of this condition will be described, as well as a number of physiological and pharmacological investigations. The results of these indicate a disorder of inhibiting mechanisms which normally control the motoneurones. The undamped discharge of the motoneurones was considered responsible for the maintenance of the severe muscular rigidity. The anatomical findings at death have been compared with the clinical, physiological, and pharmacological data.

The present case differs in certain respects from the others described in the literature. In this patient the rigidity evolved only as his neurological state deteriorated, whereas the previously reported cases were of acute onset, most of them being due to trauma. Furthermore, the distribution of rigidity in this patient was such that the resulting posture of the arms was one of adduction and extension, similar to the quadrupedal decerebrate posture, instead of the more usual abduction and flexion. In spite of the progressive nature of the pathological process (a cervical tumour), there was no detectable

* Muscular rigidity is said to be present in a limb when muscles actively resist passive movement of a joint no matter what the direction of displacement. The posture imposed on the limb by rigid muscles partly depends on the size of the opposing muscle groups, and partly on the distribution and refiex accessibility of the motoneurone discharge causing the rigidity. change in the physical signs in the upper limbs onêe the adducted extended posture had become est $\vec{A}$ blished. The legs, however, passed from normal through spastic paraparesis to spastic paralysis. ${ }_{\sigma}$

The affected muscles were paralysed, and the intense, unremitting rigidity prevented passive displacement of the arms. The rigidity was subject to sudden exacerbations or spasms which gave great pain referred to the neck region. In contrast to spasticity and Parkinsonian rigidity, the paralysis was found to be largely independent of muscle spindle activity, depending instead on the hygerexcitability of alpha motoneurones, and was thus the "alpha" type described in cats by Granit (185) and in man by Rushworth (1960). Consequentlot attempts to reduce or abolish the rigidity by inet ference with segmental reflexes were doomed to failure. The electrophysiological studies demo strated a muscular rigidity due to constantly discharging motoneurones which were at least partial $\overline{8}$ isolated from their nervous connexions, and the pathological features support this view. The rigidity was inaccessible to any form of effective treatment though certain drugs were found capab伥 of modifying it for short periods of time. Its un remitting character, even during natural sleep, and the tendency to severe exacerbations of agonizing spasms were a challenge to our therapeutic and palliative resources in this most distressing, pre longed, and painful condition.

\section{Case Report}

A.N., aged 47, was an area youth organizer. He dieed on July 29, 1960.

Early in 1957 the patient began to experience pain the back of the neck, sometimes radiating into the uppas arms. It was aggravated by coughing or straining. ORe year later, the fingers of the left hand became numb, anis six months after this the left arm was noticed to be weak when swimming, and the left leg felt awkward and causeg him to stumble occasionally. Early in 1959 he wass admitted for investigation under the care of Dr. Ritchie 
Russell. Examination then showed that he carried his head slightly tilted to the right. The cardiovascular, respiratory, genito-urinary, and alimentary systems showed no abnormality. The cranial nerves and optic fundi were normal. A left Brown-Séquard syndrome with a sensory level at $\mathrm{C} 3$ was present. The proximal muscles of the left arm were slightly wasted, without fasciculation. Gait was unsteady and Romberg's sign was positive.

Routine blood counts were normal. The Wassermann and Kahn reactions were negative. Radiographs of the skull and cervical spine showed no abnormality. Lumbar puncture revealed a complete block, and the cerebrospinal fluid contained $60 \mathrm{mg}$. of protein per $100 \mathrm{ml}$. and 3 lymphocytes per $\mathrm{mm}^{3}$. The Wassermann reaction of the cerebrospinal fiuid was negative. Myelography from the lumbar region demonstrated a complete block at the level of the fourth cervical vertebra.

On February 24, 1959, Mr. Pennybacker performed an upper cervical laminectomy and the spinal cord was found to be expanded at the $\mathrm{C} 2$ to $\mathrm{C} 3$ level to the extent of filling the whole theca. It appeared grayish on the left and the diagnosis of intramedullary tumour was not in doubt. Aspiration did not yield fluid, and no biopsy was taken. The dura was left widely open and the wound sutured.

Post-operatively, the patient's condition remained static for six months, when the right arm became increasingly numb, then weak and stiff. About one month later, numbness of the right leg appeared, and a few weeks later the weakness and stiffness of both arms suddenly and rapidly increased. The condition of the legs was unaltered. Sphincter control was normal except for very occasional dribbling if the bladder became overdistended.

In December, 1959, he was readmitted to the neurosurgical unit. Examination then showed that the cranial nerves and optic fundi remained normal. In the motor system, both arms were rigidly extended and adducted while the forearms were pronated. Voluntary movement was very weak in all muscle groups in the arms. The power in the hands was surprisingly good, but it was impossible for him to abduct the arms or flex the elbow. No fasciculation was observed. Power was almost normal in the legs but was diminished in the proximal muscles. No tendon reflexes could be elicited in the arms, but they were brisk in both legs. The abdominal reflexes were present and equal. A few beats of clonus could be elicited at the left ankle and both plantar responses were extensor. The respiratory movements were somewhat limited.

In the sensory system, the sensation of pinprick was impaired, over the entire body below $\mathrm{C} 3$ on both sides, but markedly so from $\mathrm{C} 5$ to $\mathrm{T} 1$ on the right. The sensations of hot and cold were impaired below $\mathrm{C3}$ on both sides and were totally absent in the right arm. The sensation of light touch was impaired from C3 to T3 bilaterally but was normal below this. Joint position sense was impaired distally in both arms, the right being worse than the left, and vibration sense was absent below the neck on both sides. Stereognosis was impaired in both hands.

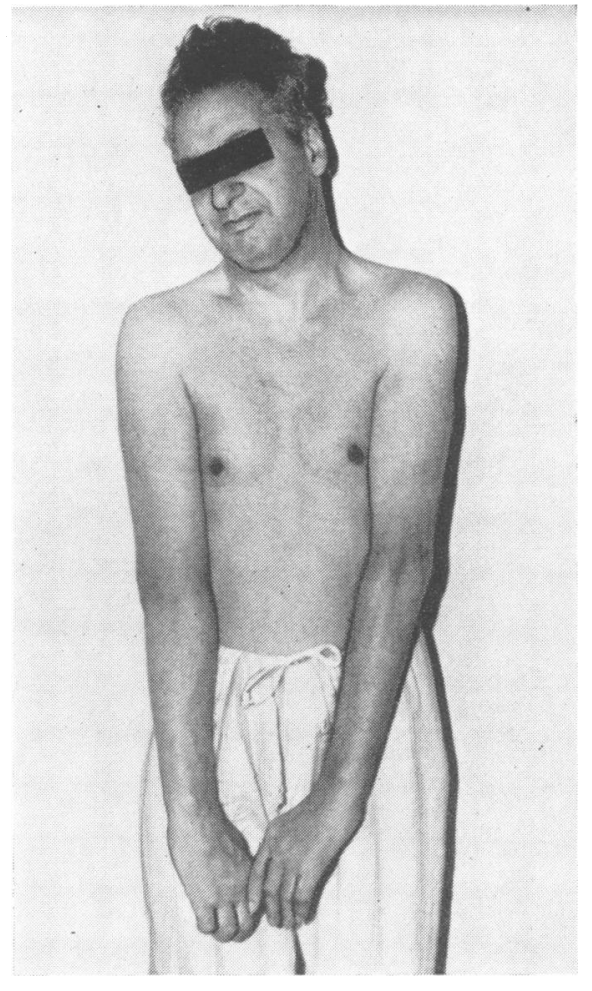

FIG. 1.-The patient standing, four months before his death, showing the fixed posture of the arms and shoulder girdle.

On December 31, 1959, Mr. Pennybacker re-operated and tumour was apparent throughout the cervical enlargement, but was greatest opposite C3, C4, and C5. A longitudinal incision $4 \mathrm{~cm}$. long was made in the left posterior column of the spinal cord and a firm pinkish mass was encountered at a depth of less than $1 \mathrm{~mm}$. This was shelled out without difficulty, except for adhesions to the white matter at the upper pole. It was an apparently circumscribed, histologically benign, astrocytic tumour weighing $2 \mathrm{~g}$. The dura was left open and the wound sutured.

Post-operatively, the weakness of the arms had increased distally, especially on the left, and the spinothalamic sensory deficit had extended to involve the whole of the right side of the body, where it was extremely dense. The condition of the legs was unchanged.

When the patient again came under the care of the neurological unit in March, 1960, there was little change in his condition, the most striking feature being the intense spasm of shoulder and arm muscles which imposed on the upper extremities the posture of adduction, internal rotation, extension, and semipronation. The shoulders were drawn forward, and the head tilted to the right (Fig. 1). In spite of a mild spastic paraparesis worse on the left, he was able to stand and to walk quite well with support. The abnormal posture of the 
arms persisted unchanged until his death from bronchopneumonia four months later. During this time movement at the fingers and wrist became progressively impaired and the muscles rigid, fixing the wrist in extension and the fingers in flexion. The spastic paraparesis gradually progressed to complete spastic paraplegia and respiratory movements became weaker.

Electrophysiological Observations.-Three months before his death simultaneous recordings of the electrical activity of, first, the right deltoid and pectoralis major muscles, then the right biceps and triceps muscles were taken using coaxial needle electrodes in the muscles and a balloon over the operative joint to signal movement there. (For details of methods see Rushworth, 1960.)

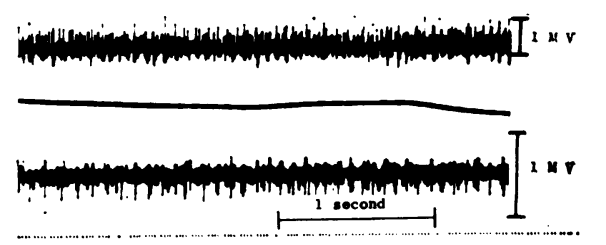

Fig. 2.-Electromyogram recordings from the right deltoid muscte (upper line) and the right pectoralis major muscle (lower ling. Mechanogram (middle line) from a balloon over the pectorats major to signal movement at shoulder joint. Spontaneoffs activity.

(a)

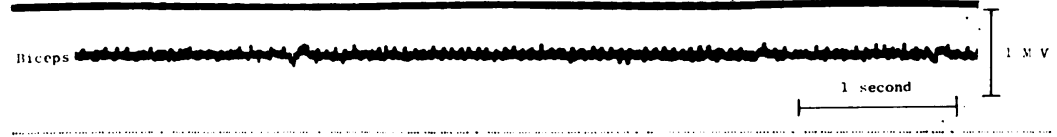

(b)

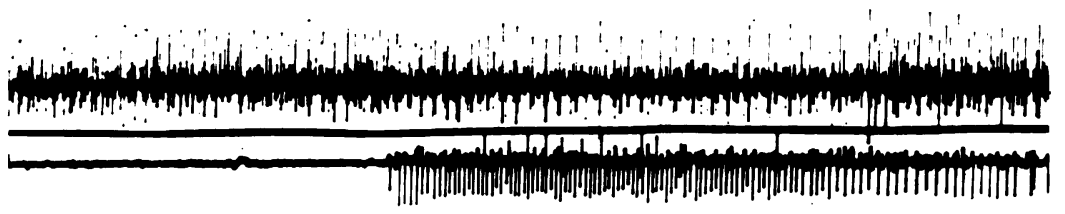

(c)

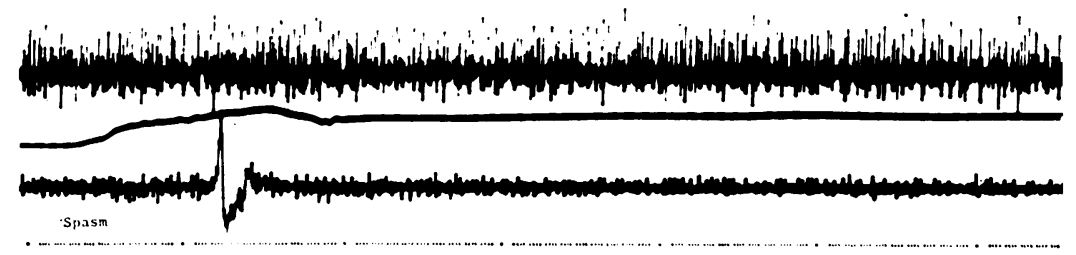

(d)

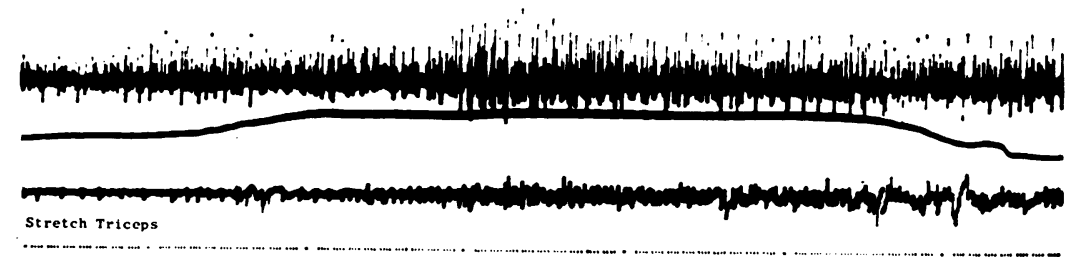

FIG. 3.-Electromyograms from the triceps (upper line) and the biceps (lower line). (a) Spontaneous activity; (b) attempt to flex arm produces single unit response in the biceps and some inhibition of the triceps discharge; (c) a spontaneous spasm evokes an increased firing rate in both triceps and biceps; (d) passive stretch of the triceps has at first little effect until a spasm in both triceps and biceps supervenes. 
Deltoid and Pectoralis Major.-The spontaneous activity in both these muscles was intense, with many motor units firing at rates up to $20 / \mathrm{sec}$. (Fig. 2). This was not influenced by painful stimulation of the skin or by passively turning the head and neck. When the pectoralis muscle was stretched by attempted passive abduction of the arms, there was a considerable increase in the electrical activity of this muscle and also in its anatagonist, the deltoid. This was similar to the stretch response described below for the triceps muscle (Fig. 3d). It was not a true stretch reflex because the muscle response did not appear as soon as the muscle was stretched but occurred suddenly during stretching, and with a simultaneous outburst in its antagonist. The burst of activity in the pectoralis muscle was so great that the limb became firmly adducted again. This activity did not cease when the muscle shortened, but slowly decreased over several minutes. In these features it resembled the spasms which occurred spontaneously from time to time.

When the patient attempted to abduct the arm there was very little increase in the motor unit discharge of the deltoid, but the pectoralis responded with an outburst of activity which was almost as great as when the patient tried to adduct the arm voluntarily.

Following a xylocaine injection around the brachial plexus by the method of Lookman (1958), the stretch response and voluntary activity were gradually blocked simultaneously. When the block was maximal it was quite easy to abduct the arm passively to $90^{\circ}$ before considerable resistence was met (presumably contracture).
Before injection $15^{\circ}$ of passive abduction always met with overwhelming resistance.

Biceps and Triceps.-A great deal of spontaneous activity was recorded in these muscles (Fig. 3a) which was greatest in the triceps muscle where several motor units were discharging at about $15 / \mathrm{sec}$. In the recordings from the biceps muscle, a small single motor unit was also discharging at about $15 / \mathrm{sec}$. This spontaneous activity was surprisingly constant for long periods of time, but there were occasional bursts of increased firing simultaneously in the two muscles associated with a spontaneous painful spasm (Fig. 3c). The discharge rate during the spasm was about twice that of the steady spontaneous activity and it took several minutes to resume the lower rate again. At no time were excessively high discharge rates observed, and it was not possible to influence the motoneurone discharge either by passively turning the head and neck, or by painful stimulation of the fingers.

In spite of the clinical impression that no voluntary activity was present in these arm muscles, the electromyogram of the biceps showed a single unit discharge when the patient was asked to try to flex his arm (Fig. 3b). During the biceps discharge, the spontaneous discharge rate in the triceps was slowed, a few motor units even being completely inhibited. The ending of the contraction of the biceps was heralded by a deceleration of the single motor unit recorded in that muscle, and by a burst of increased activity in its antagonist. No voluntary activity was seen in the triceps.

(a)

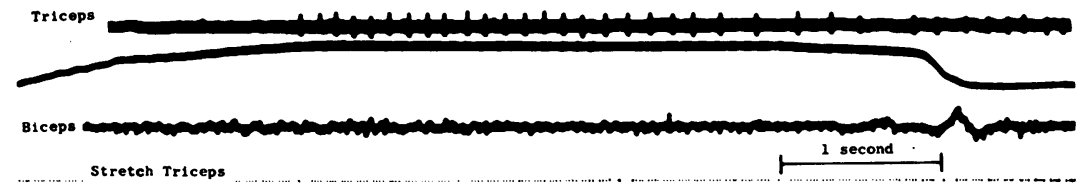

(b)

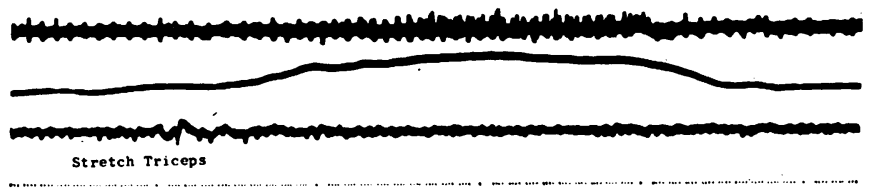

(c)

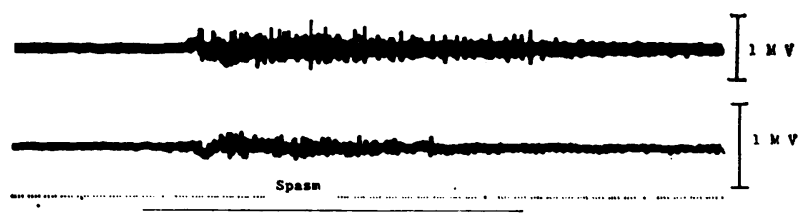

Fig. 4.-Five minutes after the procaine injection. (a) No spontaneous activity in the triceps, but a single unit is evoked by passive stretch; (b) repeated passive stretch produces a greater response, this being the fifth repeated stretch response; (c) a spontaneous spasm shows that the triceps is still innervated but much less than previously. 
Fic. 5.-Ten minutes after the procaine. injection. (a) The stretch responde of the triceps is absent and thero is no spontaneous activity; (b).a spasm shows that the triceps is now denervated by the loc

(b)

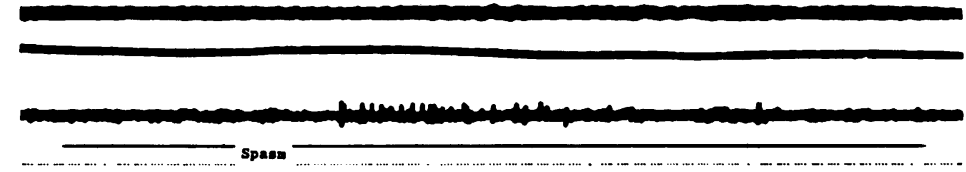

The response of the triceps muscle to slow stretch (Fig. 3d) added little at first to the spontaneous activity in this muscle, but continued stretch eventually evoked a spasm in both the triceps and biceps muscles which was not interrupted by relaxation of the stretching stimulus. There appeared, therefore, to be very little true stretch reflex in the triceps muscle.

The injection of $10 \mathrm{ml} .2 \%$ procaine near the motor points of the triceps muscle produced, almost at once, reduction of the spontaneous discharge of motor units in that muscle. Only three units fired spontaneously and the discharge rate of one of them was about $9 / \mathrm{sec}$. Spontaneous activity in the biceps continued, of course, at its previous rate. Stretching the triceps muscle produced only a small stretch reflex with increased activity also in the antagonist. The spontaneous activity of the triceps could be completely inhibited by efforts to contract the biceps muscle, which in fact could now bring about slight flexion of the arm. On ceasing the attempt to flex the arm the spontaneous activity of the triceps returned and accelerated; only two motor units were, however, active.

Five minutes after the procaine injection (Fig. 4) the spontaneous activity had completely disappeared, but by stretching the triceps muscle, a single unit response could be obtained (Fig. 4a). Repeated stretches brought back a background of spontaneous activity, and by the fifth successive stretch four motor units were responding (Fig. 4b). Presumably, therefore, the triceps motoneurones were still susceptible to post-tetanic potentiation from muscle-spindle afferents. A spontaneous spasm affecting both biceps and triceps occurred (Fig. 4c) and this showed that the triceps muscle was still partly innervated, though some motor nerve fibres were clearly blocked by procaine as the motor unit discharge was much less than before the injection.

Ten minutes after the injection of procaine (Fig. 5a) there was no spontaneous activity in the triceps and no stretch response. During a spasm, the triceps muscle no longer responded (Fig. 5b) and was now completely paralysed by the local anaesthetic.

It is apparent, therefore, that the small stretch reflex of the triceps muscle in this patient persisted until it was paralysed by the procaine. These facts make it very unlikely that hyperactive muscle spindles wey responsible for the rigid state, for they would have produced a characteristic stretch reflex which wouf begin immediately the muscle was lengthened and cease when it was allowed to shorten. Furthermore, gamma motor fibre activity, which can cause muscle spindle hyperactivity and the exaggerated stretch reflex of spasticity, is particularly sensitive to procaine and if thus blocked before other reflexes or voluntary activity (Matthews and Rushworth, 1957; Rushworth, 196). This rigidity is, therefore, of the "alpha type" (Polloc and Davis, 1931; Granit, 1955), which in animalo is unaffected by dorsal root section and is beliegeg to depend principally on the hyperexcitability the motoneurones themselves or by an imbalance of facilitatory and inhibitory mechanisms acting them.

The studies of the pectoralis and deltoid muscle showed a considerable disorder of the reflexes acting of antagonists (reciprocal innervation). The vast amount of spontaneous activity and stretch responses (which wefe no more sensitive to local anaesthetic than was the voluntary activity of the muscles) showed that the muscular rigidity was of the alpha type due to hype? excitability of the motoneurones themselves and indeperdent of local reflexes.

Few surviving motoneurones of the deltoid and biceps could be excited by voluntary effort, and they were ngt activated by painful stimulation of the skin and turnimg of the head and neck. Passive stretch of the triceps muscle had little effect on the intense spontaneogs motoneurone discharge, and voluntary effort had nonk.

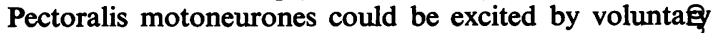
effort, but only a little by other excitatory afferents, and they could not be inhibited by attempted contraction of the antagonistic muscle. These facts lead one to believe that the intrinsic cord lesion causing these disorders was probably one interfering with the connexions of the interneurones which are so very much concerned wi the segmental interplay of reflexes as well as connected with the many supraspinal and intersegmental influencs finally inpinging on the motoneurones. 
Therapeutic Trials.-Rational means of alleviating the alpha type of rigidity will depend on methods for decreasing or removing factors which are known to stir up the motoneurones, or they will attempt to interrupt or reduce the discharge at some point in the final common pathway to the affected muscle. They are very limited, but the following procedures were tried:

Repeated passive movements were attempted but were defeated by the pain produced (in spite of analgesics) and by the intensity of the spasm at all joints other than the fingers and wrist. Heat applied by an electric blanket lessened spontaneous pain but not the rigidity. Prolonged splinting with elbows in flexion produced no benefit, and gentle traction to draw the arms away from the trunk could be tolerated for only a few minutes at a time.

A sphygmomanometer cuff was inflated to $180 \mathrm{~mm}$. mercury for 25 minutes in order to render the nerves and muscles of the limb ischaemic, and for half an hour afterwards there were fewer spontaneous spasms and finger movements were freer.

Mephanesin elixir was given orally in doses up to $1 \mathrm{~g}$. four-hourly without demonstrable effect.

Chlorpromazine was given intravenously in a dose of $50 \mathrm{mg}$. with considerable diminution in the rigidity of all muscle groups for four to five hours afterwards. Sudden passive movements of the joints still induced severe painful spasms, however. An oral dose of $150 \mathrm{mg}$. four times a day slightly decreased the rigidity, but the effect diminished after two or three days.

Sodium thiopentone (Pentothal) was given intravenously in a dose just sufficient to depress respiration, and it immediately produced considerable relaxation of rigidity, and $50 \mathrm{mg}$. of succinyldicholine chloride (Scoline) intravenously maintained the relaxation. When the effect of the Pentothal and Scoline had worn off, $90 \mathrm{mg}$. of gallamine triethiodide (Flaxedil) was given intravenously almost completely relaxing the rigidity for 30 minutes. Spasm in response to sudden movements returned before the constant rigidity, and for three hours afterwards the patient was able to tolerate flexion of the left elbow almost to a right angle.

D-tubocurarine chloride (Curare) was given intravenously in a dose of $5 \mathrm{mg}$. without general anaesthesia. This produced immediate and considerable relaxation of rigidity, and no spasms occurred for half an hour. The effect wore off gradually over the course of five hours. Ten minutes after the injection, when relaxation was maximal in the arms, it could be demonstrated that power was unaffected in the legs or trunk.

Ten millilitres of $20 \%$ magnesium sulphate solution, were injected slowly intravenously, and had no effect on the rigidity but made the patient sleepy.

Ten millilitres of $20 \%$ calcium gluconate solution were injected slowly intravenously and had no effect.

Chlorothiazide, $0.5 \mathrm{~g}$. twice daily, was given orally without potassium supplements for five days in order to lower the serum potassium level. This fell to $3.1 \mathrm{mEq}$./ litre for three days during which there was depression of the ST segments in the electrocardiogram. No effect was noted on the rigidity or spasms.

Two per cent procaine hydrochloride was infiltrated extensively into the triceps and biceps muscles of one arm with some relief of rigidity for half an hour. Infiltration of the lateral and posterior cervical muscles lessened their rigidity for several hours during which the spontaneous painful spasms of the arms were considerably reduced. Finally, procaine block of the brachial plexus by the method described by Lookman (1958) was repeatedly shown to abolish rigidity for periods up to two hours.

Thus the agents found to modify this patient's rigidity were chlorpromazine, thiopentone, depolarizing and anti-depolarizing relaxants, and procaine. Unfortunately, none acted in a way which was of significant therapeutic benefit.

Pathology.-Necropsy was performed 28 hours after death (Radcliffe Infirmary, Oxford, P.M. No. 627/60). Abnormal findings were confined to the respiratory, nervous and muscular systems.

Respiratory System.-The immediate cause of death was an extensive bilateral bronchopneumonia, obvious macroscopically and confirmed histologically.

Nervous System.-The brain weighed 1,400 g. and appeared normal externally. No abnormality was seen in centimetre coronal slices of the cerebrum, and histological sections of the cerebellum, pons, and upper medulla showed no changes. The changes in the lower medulla will be described with those of the spinal cord.

The spinal cord was removed in two pieces: an upper portion, comprising segment $\mathrm{Cl}$ to $\mathrm{T} 6$, within the vertebral column and post-vertebral muscles, and a lower portion removed in the usual way from the spinal canal, which here showed no abnormality.

The upper portion was dissected after fixation. There had been a laminectomy of the second to sixth cervical vertebrae. The overlying muscles had healed by fibrous scarring without suppuration. Deep to these muscles was a cavity, measuring $10 \mathrm{~cm}$. from above down and $3 \mathrm{~cm}$. transversely, filled with clear fluid and lined by a glistening membrane. Projecting into this cavity from the posterior aspect of the third, fourth, and fifth cervical cord segments was a soft pink tumour mass about $2 \mathrm{~cm}$. in diameter. The dura was deficient in this region. Anteriorly, the cord from C2 to C6 was flattened and expanded, especially on the left. The emerging roots and ganglia appeared normal, and the leptomeninges showed no sign of tumour infiltration. In transverse sections, the cord at level $\mathrm{Cl}$ was expanded and the normal graywhite demarcation was preserved. Segments C2 to C6 were distorted and expanded by tumour, and it was not possible to distinguish macroscopically between grey and white matter, or between nervous and tumour tissue. At $\mathrm{C7}$, the normal cord pattern re-emerged, and from T1 downwards the cord looked normal.

Histology of Spinal Cord.-Transverse blocks from each cervical segment and from the first and tenth thoracic and second lumbar segments were embedded in paraffin wax and sections stained for nerve cells, nerve fibres, myelin, and neuroglia. A block from T8 was stained by the Marchi technique. Frozen sections were 
cut transversely from $\mathrm{C} 3$ and stained to demonstrate lipid, axons, astrocytes, and microglia.

The histological appearance of the tumour differed strikingly from that of the mass removed from the posterior columns seven months before death. The earlier specimen showed no signs of malignancy; it consisted of a dense feltwork of glial fibres mixed with astrocytes and Hortega cells. Numerous small, thickwalled vessels were present, many showing dense lymphocytic cuffing (Fig. 6). No nerve cells or nerve fibres were seen.

The tumour found at necropsy was a diffuse cellular astrocytoma extending from the lower medulla (at the level of the obex) down to the eighth cervical segment. The extent of tumour infiltration is shown in Fig. 7. Infiltration was most intense in the gray matter, and at the upper and lower limits of the affected region it was practically confined to the gray horns. The tumour showed regional differences; parts were pilocytic, others fibrillar, others gemistocytic (Fig. 8). In the infiltrated gray matter, surviving neurones were seen, and in the white matter, although myelin had disappeared, there were numerous axons threading their way between the tumour cells.

An area of malignant change was present on the left, extending between the segments $\mathrm{C} 2$ and $\mathrm{C6}$. The appearance at $\mathrm{C2}$ is shown in Fig. 9. Here the picture was that of a pleomorphic glioblastoma, with giant cells (Fig. 10). Tumour had destroyed and replaced the left posterior horn and posterior and lateral columns, and by its expansion had produced severe distortion of the remaining cord.

The myelin and Marchi stains showed Wallerian degeneration corresponding to a cord transection complete on the left side, incomplete on the right. The effects were seen in the lower medulla and $\mathrm{Cl}$ segment as degeneration of the cuneate and gracile tracts and of the left spinocerebellar tracts. Below the tumour, the pyramidal tracts were degenerate, the left one completely so.

Cervical Cord Neurones.-The pattern of cell loss and cell damage in the anterior horns and intermediate gray matter is of especial interest in this case. Unhappily, the estimation of these features is to a large extent subjective. Whereas large motor cells may be readily recognized and their severe depletion be apparent, it is less easy to recognize moderate cell loss in a grossly distorted cord infiltrated with tumour. As to the smaller nerve cells, it is often impossible to decide whether their appearance is normal or pathological; whether, for instance, a particular cell should be described as "small and dark" or "shrunken and pyknotic". In some areas, even the distinction between neurone and astrocyte is doubtful. The description which follows should be read with these reservations in mind.

In sections from the lower medulla, the nerve cells of hypoglossal and supraspinal nuclei appeared intact, in spite of slight tumour infiltration. Motor cell loss was first apparent in the lower part of the first cervical segment, the loss being greatest on the right. Both anterior horns were grossly depleted from $\mathrm{C} 2$ to $\mathrm{C6}$, and no motor cells were seen on the left in segments C4 an C5. From C7 downwards the anterior horns containe 9 motor cells in normal numbers (see Table).

TABLE

EXTENT OF MOTOR CELL LOSS IN SEGMENTS OF CERVICAL CORD

\begin{tabular}{|c|c|c|}
\hline Level & Right & Left \\
\hline $\begin{array}{l}\text { C1 } \\
\text { C2 } \\
\text { C3 } \\
\text { C4 } \\
\text { C5 } \\
\text { C6 } \\
\text { C7 } \\
\text { C8 }\end{array}$ & $\begin{array}{l}\text { Slight } \\
\text { Severe } \\
\text { Severe } \\
\text { Severe } \\
\text { Severe } \\
\text { Slight } \\
\text { None } \\
\text { None }\end{array}$ & $\begin{array}{l}\text { Slight } \\
\text { Severe } \\
\text { Severe } \\
\text { Total } \\
\text { Total } \\
\text { Severe } \\
\text { Slight } \\
\text { None }\end{array}$ \\
\hline
\end{tabular}

The smaller nerve cells of the intermediate and posterior gray matter could be distinguished in the upper and lower cervical segments on both sides. In the more्E affected segments, they were reduced in number op absent but the presence of tumour made reliable counts impossible.

The morphology of the motor cells in the tumouris infiltrated horns was abnormal. The most striking change was a rounding-off of the cell outline, and losis. of dendrites. The remaining dendrites were usuallf tenuous and they stained feebly (Figs. 11 to 15). This change seemed significant, as the motor cells of the hypoglossal nucleus and of the anterior horns betow the tumour showed normal antler-like expansions. 응

Spinal Nerve Roots. Posterior Root Ganglia, Peripher.a Nerve, and Muscle.-The anterior roots showed losforo fibres corresponding to the loss of cells in the cervical cord segments, as described above. The posterior root and their ganglia showed no change. The tumour has not extended along the nerve roots.

Silver impregnation of peripheral nerve supplying muscles of the upper limbs showed variable numbers of axons undergoing fragmentation.

The following muscles were examined: Tongue, righ and left sternomastoid (C1-5), diaphragm (C3-5), righ and left deltoid (C5-6), right and left pectoralis major (C5-T1), left biceps brachii (C5-6), right palmaris longus. (C8), right and left lumbricals (C8-T1), and rectus abdominis.

As might be expected, the tongue and rectus abdomin showed no abnormalities. All the other muscles examined showed atrophy indicative of recent partial denervatio (Fig. 16). Many of them also contained scattered inter? fascicular inflammatory foci, consisting of lymphocytes? neutrophils, and monocytes (Fig. 17). These inflame matory changes are most plausibly attributed to mechanical damage due to prolonged spasm.

In relating the pathological to the clinical features of this case, it has to be remembered that the pathor logical description refers to the patient's final state whereas most of the physiological observations refer to a period four months earlier. It is not known when the spinal tumour underwent malignant 


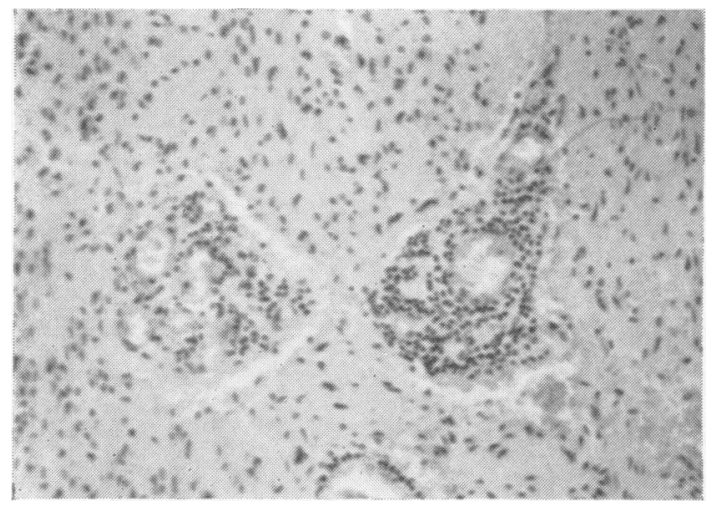

Fig. 6. Section of intramedullary astrocytoma removed seven months before death. Similar astrocytic and vascular profileration was present in areas of the tumour examined at necrospy. Haematoxylin and eosin $\times 133$.
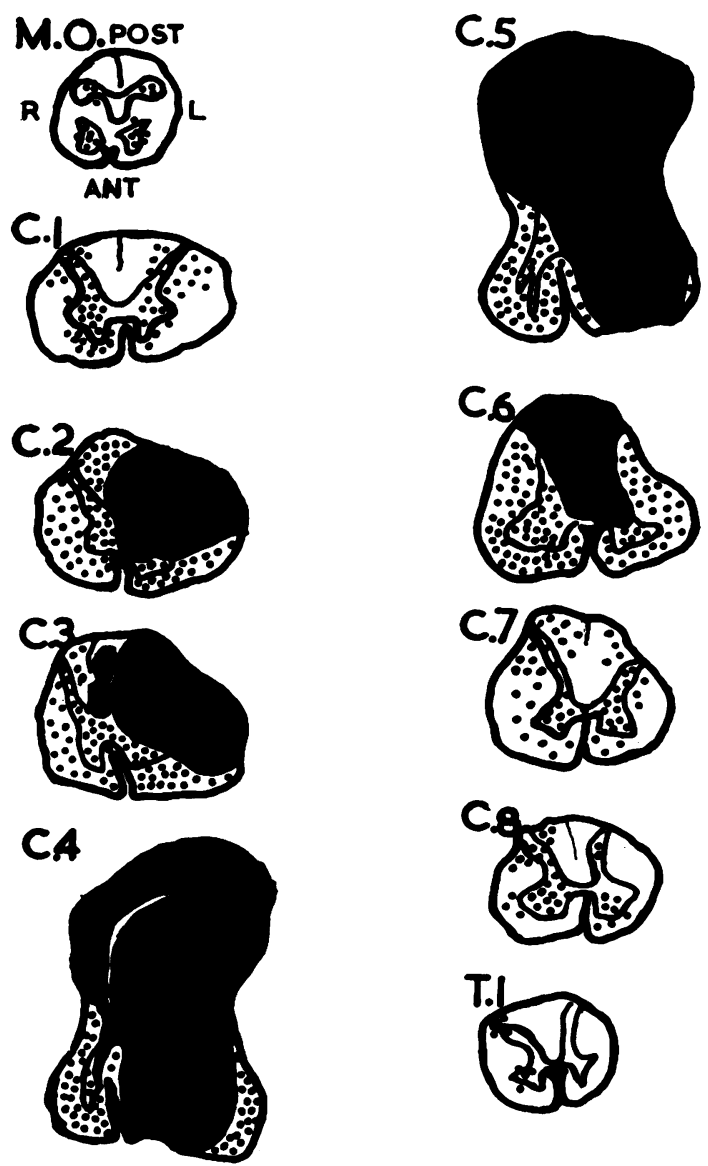

FIG. 7.-Diagrams from camera lucida tracings of histological preparations illustrating the changes in the cervical cord at successive levels. Stippling indicates infiltration of nervous tissue by astrocytoma. Solid black indicates destruction of nervous tissue and replacement by malignant tumour $\times 2$. Seen from upper surface; the left side of the cord is on the right of each diagram.

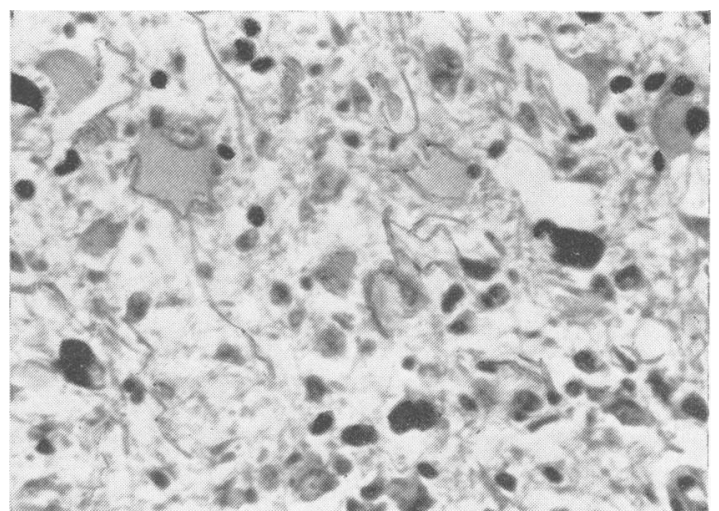

Fig. 8.-Transverse section of spinal cord segment C4 showing gemistocytic astrocytoma. Phosphotungstic acid haematoxylin $\times 310$.

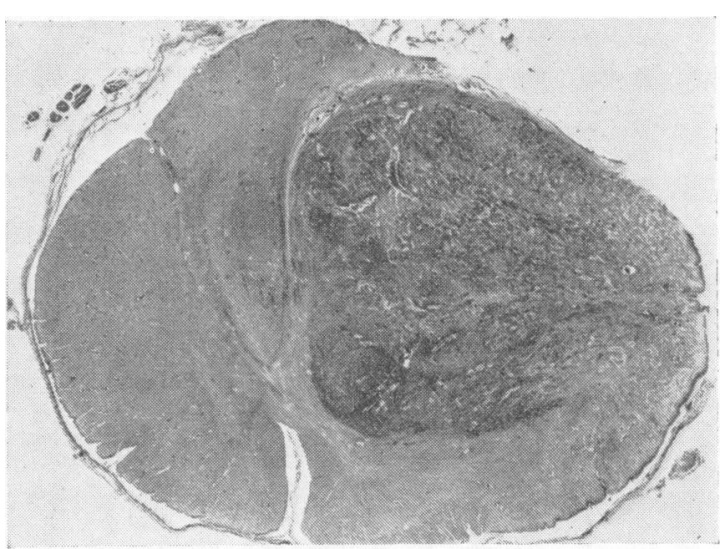

FIG. 9.-Transverse section of spinal cord segment C2 showing malignant tumour (glioblastoma) on the left. The remaining cord here is infiltrated with astrocytoma. Holmes $\times 4$.

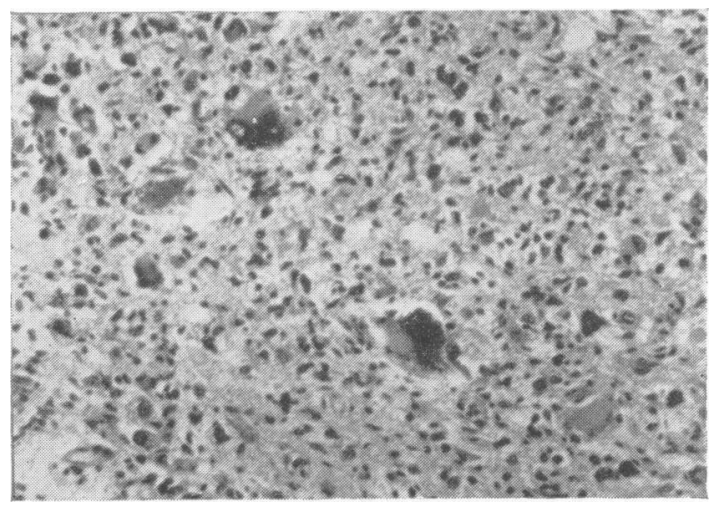

FIG. 10.-T.S. of $\mathrm{C4}$ showing malignant tumour (glioblastoma). Haematoxylin and eosin $\times 133$. 


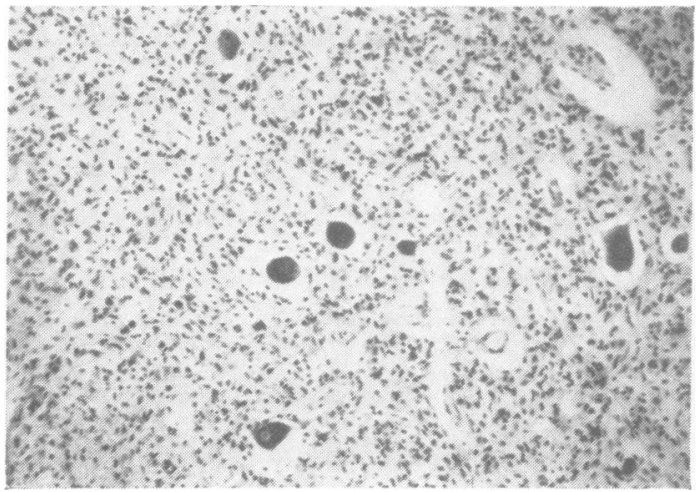

FIG. 11.-T.S. of C6 showing astrocytoma surrounding survitin motoneurones in the anterior horn. Nissl. $\times 80$.

FIG. 12.-Motoneurone from anterior horn of $\mathrm{C} 2$. Note roundifg $\mathrm{o}$ contour and absence of dendrites. Glees. $\times 310$.

Fig. 13.-Normal motoneurone from C1. Glees. $\times 310$.

FIG. 14.-Motoneurone from anterior horn of C6. Note frail dend商e Nissl. $\times 310$.

FIG. 15.-Normal motoneurone from T1. Nissl. $\times 310$.

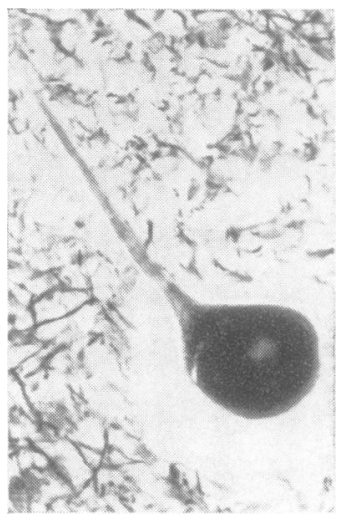

FIG. 12

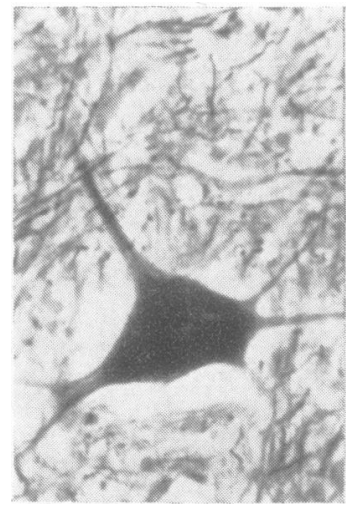

Fig. 13

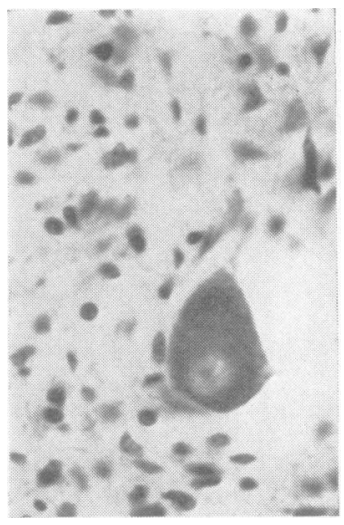

Fig. 14

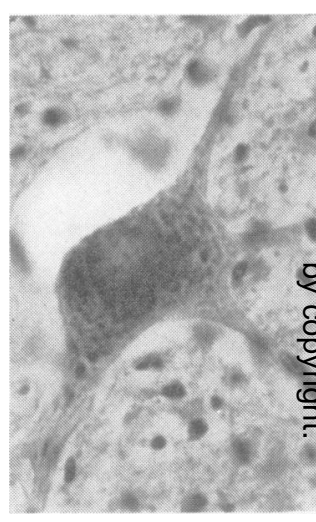

Fig. 15

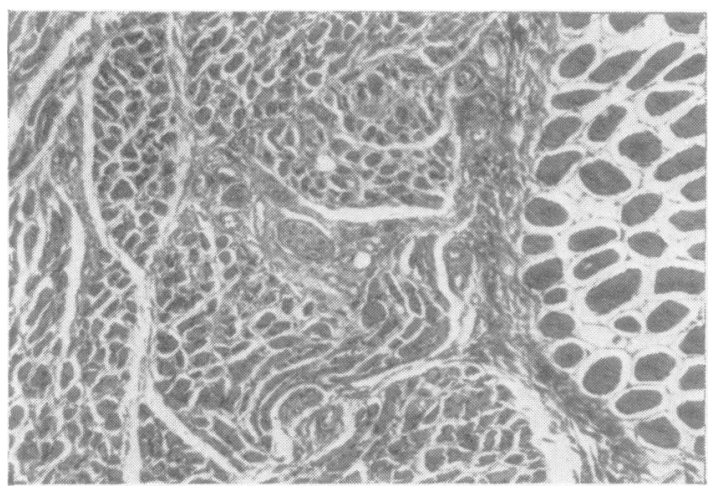

Fig. 16.-T.S. of right palmaris longus showing muscle atrophy. Haematoxylin and Van Gieson. $\times 50$.

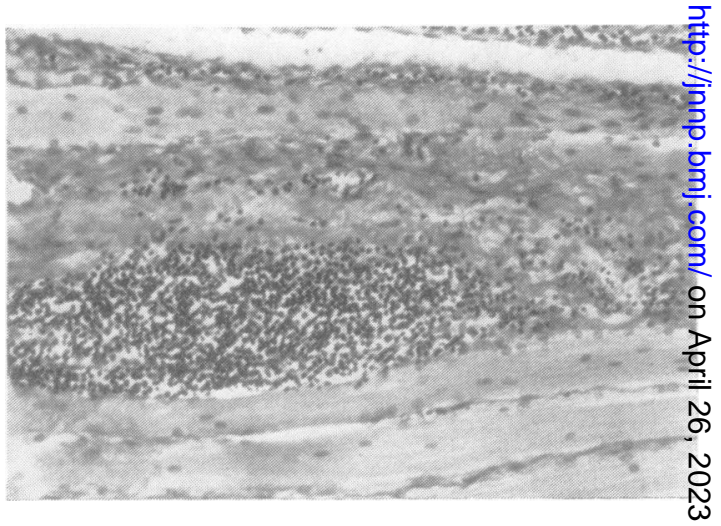

FIG. 17.-L.S. of a lumbrical muscle showing marked inflammatory reaction. Haematoxylin and Van Giesen. $\times 80$. 
change; but it is reasonable to suppose that the patient's muscular symptoms were due to the relatively slow infiltration of the anterior horns by astrocytoma, isolating without destroying the motor cells, and that the massive destructive lesion seen in segments 4 to 5 represents a late malignant development, which would ultimately substitute a flaccid paralysis for the previous muscular overactivity.

\section{Discussion}

Thorburn (1887) first clearly described the characteristic abnormal posture of the upper limbs which may result from trauma of the cervical spinal cord. Since then, this posture of rigid abduction and external rotation of the arms, with flexion of the forearms and drawing forward of the shoulders, has been recognized as a sign of severe cord injury at about the C6 segment. It has often been attributed to the unopposed activity of unaffected muscles innervated by the C5 segment but this explanation has been challenged by Penry et al. (1960).

In our own case, the posture of the arms was similar to the decerebrate posture, the arms being adducted and extended. Although there was some voluntary activity in the pectoralis major, it was the large amount of spontaneous activity which kept the arms in the adducted posture. The extension of the arms was similarly maintained by the intense spontaneous activity of extensor motoneurones, such as those of the triceps, over which there was no voluntary control. It seems probable, therefore, that it is the intensity and distribution of the spontaneous activity of motoneurones giving rise to muscular rigidity which finally determines the posture.

Spasticity and Parkinsonian rigidity in man are disorders of the stretch reflex mechanisms, and the basic defect lies in the overactivity of the motor supply of the muscle spindles ("gamma rigidity") (Rushworth, 1960). As a consequence, the afferent discharge in response to stretch is out of proportion to the stimulus and a large stretch reflex results. This can be abolished by anaesthetizing the gamma motor fibres by injecting procaine intramuscularly or partial nerve block, for these fibres are particularly sensitive to local anaesthetic. Among 50 cases, Rushworth found only one spastic case which did not fit into this category. In this case, the stretch reflex could not be selectively reduced by procaine but persisted until the muscles themselves were paralysed. In these features the spasticity resembled the alpha rigidity of the anaemically decerebrated cat (Granit, 1955) which is not abolished by dorsal root section (Pollock and Davis, 1931) or local anaesthesia (Matthews and Rushworth, 1957; Matthews, 1958). The present case was demon- strated to be an example of alpha rigidity in man. Henatsch and Ingvar (1956) demonstrated that large doses of intravenous chlorpromazine reduced the gamma rigidity of the classical decerebrate cat but had no effect on the alpha rigidity of the anaemically decerebrate cat. In man, chlorpromazine has no beneficial effect on the "gamma-rigidities" of Parkinsonsim and spasticity, and it was therefore most interesting to see some reduction of rigidity by intravenous chlorpromazine in our present case of alpha rigidity. There appears, therefore, to be a complete paradox in the actions of this drug in the cat and in man.

Intense muscular rigidity as a result of intrinsic damage to the spinal cord has been produced experimentally in cats by van Harreveld and Marmont (1939), Krogh (1950), and more recently by Gelfan and Tarlov (1959) in dogs. The latter workers temporarily occluded the high thoracic aorta for about 50 minutes, thus rendering the lumbo-sacral cord ischaemic. Severe rigidity of the hind limbs appeared as soon as the anaesthetic had worn off, and it resembled decerebrate rigidity in being most intense in the antigravity muscles. It differed, however, from decerebrate rigidity in being independent of the position or activity of the animal, and could only be abolished by anaesthesia. By analysing dorsal and ventral root potentials in response to nerve or root stimulation, they were able to show that the motoneurones lacked both inhibitory and excitatory internuncial activity and that the muscular rigidity depended largely on the continuous and spontaneous discharge of motoneurones. At necropsy, sections of the spinal cord showed destruction of the central gray matter of the cord, the anterior horns being relatively spared (Fig. 18). Our human case shows a very similar disorder of function to that described by Gelfan and Tarlov in their dogs. Reflex inhibition of

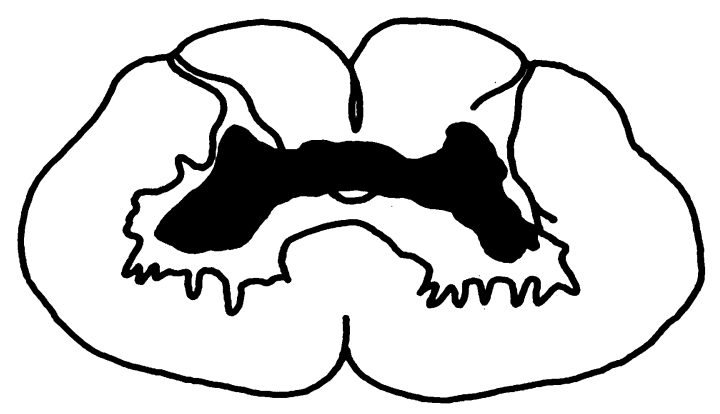

Fig. 18.-Drawing from Gelfan and Tarlov (1959) of the extent of the lesion in the lumbar cord in one of their experimental animals which during life had rigid hind limbs. (With the permission of the authors and of the editors of the Journal of Physiology.) 
samples of surviving motoneurones such as those of the pectoralis major, the deltoid, and the triceps was absent, much reduced or even reversed, and excitation of these motoneurones either by muscle stretch by turning the head and neck, by noxious stimulation of the skin, or by volition, was small or absent. The motoneurones, therefore, were partially isolated from inhibitory and facilitatory connexions, yet the severe muscular rigidity was maintained by their constant firing and even spontaneously exacerbated.

Apart from the interplay of inhibitory and excitatory afferents on the motoneurone, a continuous discharge of the motoneurone is normally held in check by a self-induced inhibitory mechanism which employs special internuncial (Renshaw) cells (Renshaw, 1941). The motoneurones, by their axon collaterals, excite the Renshaw cells, which in turn inhibit motoneurones; the distribution of this inhibitory action is said to be non-specific and indiscriminate, and Eccles (1957) believes it can only serve as an "anticonvulsive" device. It has been suggested that strychnine and tetanus toxin block the Renshaw cell mechanism and the resulting muscular convulsions are due to the very high discharge rate of motoneurones released from this inhibitory control. The continuous muscular rigidity of our patient and the spontaneous spasms may have been due to interference with the Renshaw cell system, but very high rates of motoneurone discharge were not observed.

In the present case there are two separate features of the disordered physiology which need explaining: (1) The continuous and apparently spontaneous discharge of the motoneurones maintaining the rigidity; and (2) the inaccessibility of the motoneurone discharge to a number of reflex mechanisms and to volition. The latter constitutes a functional isolation of motoneurones; to correspond with this the histological sections show motoneurones surrounded by tumour cells and an extreme lack of ramifying nerve processes in the anterior horns.

It may be that motoneurones which are isolated from their nervous connexions but are otherwise normal will discharge spontaneously, as Gelfan and Tarlov (1959) suggested. These authors remind us that both denervated muscle fibres and denervated sympathetic ganglia discharge spontaneously, and they propose that "denervated" motoneurones behave similarly. However, the isolation of moto- neurones might well produce changes in the ce which would result in spontaneous activity. In the present case, surviving motor nerve cells have rounding off of their cell bodies and a lack of dendrites. The normal surface ratio is therebeg reduced and this might conceivably result in spon taneous activity. Unfortunately, the pathologican studies do not allow us to decide between the two hypotheses of the origin of the spontaneous activity for the motoneurones were both isolated and had af abnormal morphology.

\section{Summary}

A patient with a cervical cord tumour develope intense, unremitting muscular rigidity of the arms? imposing on them a symmetrical posture similar to that of the decerebrate quadruped.

The rigidity was found electrophysiologically t $\bar{s}$ be that of the "alpha" type. The motoneurones" discharged spontaneously and were little influence by reflex mechanisms or by voluntary effort.

The cervical cord contained an infiltrating malignant glioma, causing loss of nerve cells and fibres between $\mathrm{C} 2$ and $\mathrm{C} 6$.

The architecture of the anterior horns was diß ordered, leaving surviving motor cells of which whe morphology was abnormal.

It is concluded that functional isolation motoneurones can give rise to their spontanegus discharge and muscular rigidity.

We are indebted to Dr. W. Ritchie Russell for h interest, encouragement, and advice at all stages of th work. Drs. S. Gelfan, I. Tarlov, and the Editors of the Journal of Physiology very kindly gave their permissio to reproduce Fig. 18 and we are most grateful to them.@

\section{REFERENCES}

Eccles, J. C. (1957). The Physiology of Nerve Cells. Oxford Universi Press, London.

Gelfan, S., and Tarlov, I. M. (1959), J. Physiol (Lond), 146, 594. Granit, R. (1955). Receptors and Sensory Reception. Yale University Pranit, R. New Haven.

Harreveld, A. van, and Marmont, G. (1939). J. Neurophysiol., 101. A. van, and Marmont, G. (1939). J. Neurophysiol.,

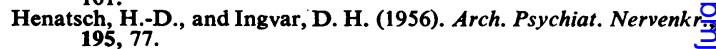

Krogh, E.'(1950). Acta physiol. scand., 20, 263.

Lookman, A. A. (1958). Anaesthesia, 13, 5.

Matthews, P.B.C. (1958). J. Physiol. (Lond.), 140, 408.

, and Rushworth, G. (1957). Ibid, 135, 245.

Penry, K. J., Hoefnagel, D., van den Noort, S., and Denny-Browm D. (1960). Arch. Neurol. (Chicago), 3, 500.

Pollock, L. J., and Davis, L. (1931). Amer. J. Physiol., 98, 47.

Pollock, L. J., and Davis, L. (1931). Amer. J.

Rushworth, G. (1960). J. Neurol. Neurosurg. Psychiat., 23, 99.

Thorburn. 'W. (1887). Brain, 9, 510. 